\title{
Development of electrochemical methods for determination of tramadol - analytical application to pharmaceutical dosage forms
}

\author{
E.M.P.J. Garrido, J.M.P.J. Garrido, F. Borges, C. Delerue-Matos
}

\begin{abstract}
A square-wave voltammetric (SWV) method and a flow injection analysis system with amperometric detection were developed for the determination of tramadol hydrochloride. The SWV method enables the determination of tramadol over the concentration range of $15-75 \mathrm{mM}$ with a detection limit of $2.2 \mathrm{mM}$. Tramadol could be determined in concentrations between 9 and $50 \mathrm{mM}$ at a sampling rate of $90 \mathrm{~h}^{-1}$, with a detection limit of $1.7 \mathrm{mM}$ using the flow injection system. The electrochemical methods developed were successfully applied to the determination of tramadol in

pharmaceutical dosage forms, without any pre-treatment of the samples. Recovery trials were performed to assess the accuracy of the results; the values were between 97 and 102\% for both methods.
\end{abstract}

Keywords: Tramadol; Square-wave voltammetry; FIA; Electrochemical detection

\section{Introduction}

Tramadol is a synthetic centrally acting analgesic agent with two distinct, synergistic mechanisms of action (Fig. 1). It is both a weak opioid agonist with selectivity for the m-receptor and a weak inhibitor of the reuptake of noradrenaline and serotonin [1]. In several controlled clinical studies, oral and parenteral tramadol effectively relieved moderate to severe postoperative pain associated with surgery. Its overall analgesic efficacy was comparable to that achieved using equianalgesic doses of morphine or alfentanil [1].

The excellent efficacy of tramadol for the management of moderate to severe pain associated with no clinically significant effects on respiratory or cardiovascular parameters, unlike other opioids, and lower potential for producing abuse or dependence led to the appearance of several pharmaceutical dosage forms in the market. Nevertheless few methodologies has been proposed for its determination, which could be related with the fact of being a relatively new drug and not 
yet included in the pharmacopoeias. Most of the analytical methods available in literature are based in chromatographic procedures, such as HPLC and capillary electrophoresis, being used to quantify tramadol in different biological fluids, namely urine, plasma and whole blood samples [2-5]. For determination of tramadol in pharmaceutical dosage forms few analytical methodologies were proposed and are mainly based in spectrophotometry [6,7], HPLC [8], capillary isotachophoresis [9] and potentiometry [10].

Although electrochemical methods have already proved to be very useful in the field of drug analysis due to their simplicity, low cost and relatively short analysis time when compared with other techniques, any electroanalytical method have been proposed for the determination of tramadol in pharmaceutical dosage forms.

An investigation has been performed on the electrochemical behaviour of tramadol at a glassy carbon electrode. The results were applied in the development of a square-wave voltammetric (SWV) method for determination of tramadol in pharmaceutical dosage forms. In order to set up an analytical procedure that could be easily used in the quality control, a flow injection analysis manifold with amperometric detection was also established.

\section{Experimental}

\subsection{Apparatus}

Voltammetric measurements were performed on a 663 VA Metrohm system containing a glassy carbon working electrode (Metrohm, $d=2.0 \mathrm{~mm}$ ), a glassy carbon rod counter electrode (Metrohm) and a $\mathrm{Ag} / \mathrm{AgCl}(3 \mathrm{M} \mathrm{KCl})$ reference electrode (Metrohm) attached to an Autolab PSTAT 10

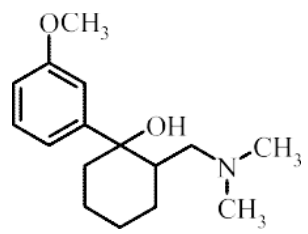

Fig. 1. Chemical structure of Tramadol.

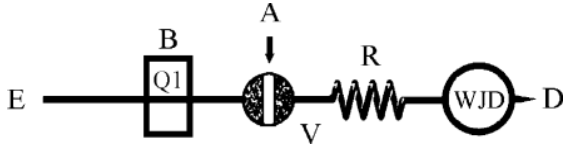

Fig. 2. Flow injection analysis manifold used for tramadol determination: A, sample; B, peristaltic pump; E; inlet for borate buffer support electrolyte; $Q 1$, flow rate $\left(2.4 \mathrm{ml} \mathrm{min}^{-1}\right)$; $\mathrm{V}$, injection valve $(80 \mathrm{ml}) ; \mathrm{R}$, mixing coil $(35 \mathrm{~cm})$; WJD, wall-jet electrochemical detector; $\mathrm{D}$, outlet for waste.

potentiostat/galvanostat running with model GPES version 3 software (EcoChimie, Netherlands).

The FIA system components were arranged as shown schematically in Fig. 2. A Gilson Minipuls 3 peristaltic pump equipped with Gilson PVC pumping tubes propelled the solutions. The pump was connected to a Rheodyne 5020 valve used to inject the solutions. An electrochemical system consisting of a VA 641 Metrohm potentiostat and a 656 Metrohm electrochemical wall-jet cell was used as the detector. The cell is composed by three electrodes, a Metrohm glassy carbon electrode as the working electrode, a Metrohm gold electrode as the auxiliary electrode and a Metrohm $\mathrm{Ag} / \mathrm{AgCl}(3 \mathrm{M} \mathrm{KCl})$ as the reference electrode. Omnifit Teflon tubing (0.8-mm i.d.) and Gilson end fittings as well as home-made dampers drilled from perspex [11] were used to connect the different manifold components. The analytical signals were recorded in a Kipp and Zonen (model BD 112) strip chart recorder.

The glassy carbon working electrodes used were manually cleaned and polished every day with an abrasive surface, first with $\mathrm{Al}_{2} \mathrm{O}_{3}(\mathrm{BDH})$ and then with water only. After polishing, the electrode surface was thoroughly washed with deionised water.

A Metrohm E520 pH-meter and a glass electrode were used for the $\mathrm{pH}$ measurements.

\subsection{Reagents and solutions}

All chemicals used were of analytical reagent grade and were employed without further purification. Deionised water with a specific conductance less than $0.1 \mathrm{mS} \mathrm{cm}^{-1}$ was used throughout.

Buffer solutions used in voltammetric studies were prepared as described elsewhere [12]. 
Borate buffer $(\mathrm{pH} 9.3 ; 0.1$ M) solution was prepared diluting $500 \mathrm{ml}$ of $0.025 \mathrm{M}$ sodium borate (Merck) and $30 \mathrm{ml}$ of $0.1 \mathrm{M}$ sodium hydroxide (Merck) in 11 of water.

Tramadol hydrochloride was kindly provided by Tecnifar (Lisbon, Portugal); dosage forms containing tramadol were purchased from commercial sources.

\subsection{General procedure}

\subsubsection{Square-wave voltammetric method}

A $2.4 \mathrm{mM}$ stock standard solution of tramadol hydrochloride was prepared in deionised water. Portions (60-300 ml) of this solution, accurately measured, were added at electrochemical cell to 10 $\mathrm{ml}$ of borate buffer $(\mathrm{pH} \mathrm{9.3;0.1} \mathrm{M).} \mathrm{The} \mathrm{peak}$ current obtained was measured and plotted against the concentration of tramadol to obtain the standard calibration graph. The same procedure was followed for sample analysis.

\subsubsection{Flow injection method}

A stock standard solution $(2.4 \mathrm{mM})$ of tramadol hydrochloride was prepared. Five standard solutions, between 9 and $50 \mathrm{mM}$, were prepared by careful dilution of the stock solution with the borate buffer supporting electrolyte. These solutions were inserted, via injection valve, in the FIA system and the peak heights obtained were measured and plotted against the concentration of tramadol to obtain the standard calibration graph. All these solutions were prepared daily. The same procedure was followed for sample analysis.

\subsubsection{Sample preparation}

The determination of tramadol was performed in different formulations (capsules, tablets, ampoules and oral solution) that are commercially available in Portugal.

The content of 10 capsules or tablets were finely powdered and thoroughly mixed. An accurately weighed amount of powder equivalent to $50 \mathrm{mg}$ of tramadol hydrochloride was dissolved in the supporting electrolyte solution ( $\mathrm{pH}$ 9.3). Filtration of solution through Whatman No. 42 filter was performed to remove any remaining insoluble matter.
The content of three ampoules was mixed and an aliquot of the solution equivalent to $50 \mathrm{mg}$ of tramadol hydrochloride was quantitatively diluted with the supporting electrolyte solution $(\mathrm{pH}$ 9.3). Oral solution was simply diluted with the same electrolyte.

In all cases, working solutions of the pharmaceutical formulations were prepared exactly as the standard solutions.

\subsubsection{Validation of the analytical methods}

Once the square-wave and flow injection experimental conditions were established validation of the methods was performed.

The linearity of the methods was determined by building five-point calibration curves in the range 15-75 $\mathrm{mM}$ and 9-50 $\mathrm{mM}$ tramadol concentrations for voltammetric and flow injection method, respectively.

The limit of quantification (LOQ) and the limit of detection (LOD) were calculated according to USP 25 guidelines [13]. A $\mathrm{S} / \mathrm{N}$ ratio of ten and three was used, respectively.

Solutions extracted from formulations at nominal concentrations of 15,45 and $75 \mathrm{mM}$ and 9, 30 and $50 \mathrm{mM}$ were analysed by the square-wave and FIA method, respectively. These assays were repeated five times in the same day to obtain repeatability values and five times over three different days to obtain intermediate precision values.

In order to verify the accuracy of the method, recovery assays at three different concentrations were carried out by adding to the formulations known amounts of tramadol powder. The final nominal concentrations of tramadol were 30,50 and $75 \mathrm{mM}$ for the voltammetric method (i.e. additions of 15,35 and $60 \mathrm{mM}$ to a formulation solution with a nominal tramadol concentration of $15 \mathrm{mM}$ ) and 19, 35 and $50 \mathrm{mM}$ for the flow injection method (i.e. additions of 9, 25 and 40 $\mathrm{mM}$ to a formulation solution with a nominal tramadol concentration of $10 \mathrm{mM}$ ). These assays were repeated five times over three different days to obtain intermediate precision data. 


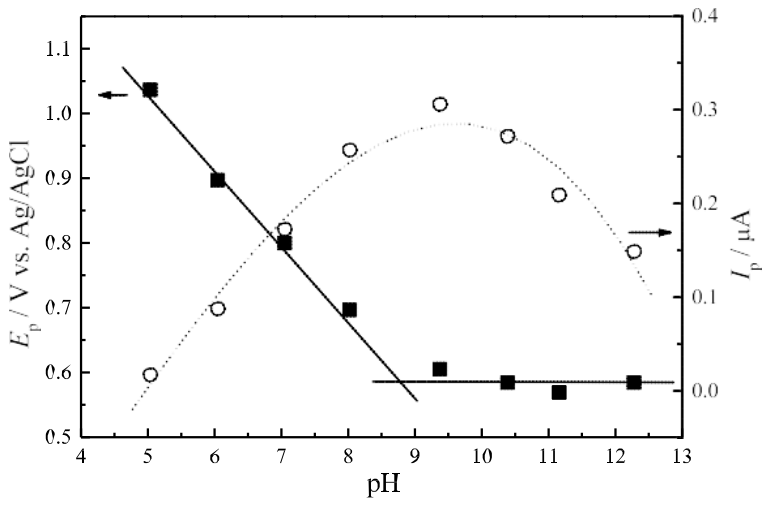

Fig. 3. Plots of $E_{\mathrm{p}}$ and $I_{\mathrm{p}}$ vs. $\mathrm{pH}$ from differential pulse voltammograms of $0.1 \mathrm{mM}$ solutions of tramadol in $0.1 \mathrm{M}$ ionic strength buffer electrolyte. Scan rate $5 \mathrm{mV} \mathrm{s}^{-1}$.

\section{Results and discussion}

\subsection{Influence of $p H$ of supporting electrolyte}

Differential pulse voltammetric behaviour of tramadol hydrochloride $(0.1 \mathrm{mM})$ at a glassy carbon electrode was examined varying $\mathrm{pH}$ over a wide range of values from acidic to alkaline media (between 1.2 and 12.8). This study led to conclude that tramadol is electroactive and that the mechanism of oxidation is dependent of $\mathrm{pH}$. For pHs lower than 4 no oxidation peak is noticeable; above that value a peak is observable at $E_{\mathrm{p}}=+1.0 \mathrm{~V}$ vs. $\mathrm{Ag} / \mathrm{AgCl}$. The shift in the differential pulse peak potential as a function of $\mathrm{pH}$ was studied and a linear dependence was observed until pH 9.3 (Fig. 3). For $\mathrm{pH}$ higher than 9.3 no variation in the peak potential was detected. The influence of $\mathrm{pH}$ on the peak current of tramadol was also investigated. Fig. 3 shows the dependence of the peak current on $\mathrm{pH}$. It is possible to observe a peak current increase until $\mathrm{pH} 9.3$ followed by a decrease above this $\mathrm{pH}$. The optimum $\mathrm{pH}$ adopted for further studies was, therefore, 9.3 .

\subsection{Development of the $S W V$ method}

The experimental parameters in SWV are interrelated and have a combined influence on the peak current. Hence, in order to establish the optimum

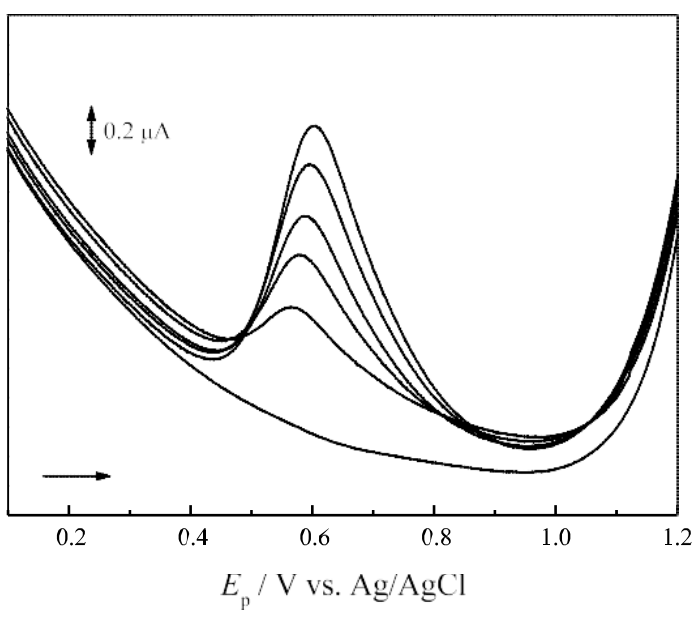

Fig. 4. Successive square-wave voltamograms in $\mathrm{pH} 9.30 .1 \mathrm{M}$ buffer electrolyte of tramadol: 0, 14.4, 28.8, 43.2, 57.6 and 72.0 $\mathrm{mM}$; frequency $50 \mathrm{~Hz}$; pulse amplitude $50 \mathrm{mV}$.

conditions for the determination of tramadol, the influence of parameters, such as frequency, $f$, and pulse amplitude, $E \mathrm{~s}$, on the current response was studied.

The square-wave frequency was varied from 10 to $200 \mathrm{~Hz}$. The peak current increased with frequency, however above $50 \mathrm{~Hz}$ a decrease in peak definition is also observed. Hence this

Table 1

Analytical parameters for SWV method and flow injection analysis with electrochemical detection (FIA-EC)

\begin{tabular}{lll}
\hline Parameter & $\begin{array}{l}\text { Method } \\
\text { SWV }\end{array}$ & FIA-EC \\
& & \\
\hline $\begin{array}{l}\text { Concentration range }(\mathrm{mM}) \\
\text { Calibration graph }(n=5)\end{array}$ & $15-75$ & $9-50$ \\
Intercept (mA) & 0.10 & 0.019 \\
Slope (mA mM $\left.{ }^{-1}\right)$ & 15.5 & 4.90 \\
Correlation coefficient & 0.995 & 0.999 \\
Standard error of intercept & 0.15 & 0.03 \\
Standard error of slope & 0.1 & 0.04 \\
LOD (mM) & 2.2 & 1.7 \\
LOQ (mM) & 7.3 & 5.7 \\
Intra-day precision (RSD\%) & $15 \mathrm{mM}(2.3)$ & $9 \mathrm{mM}(1.2)$ \\
& $45 \mathrm{mM}(1.8)$ & $30 \mathrm{mM}(0.8)$ \\
& $75 \mathrm{mM}(1.4)$ & $50 \mathrm{mM}(0.6)$ \\
Inter-day precision (RSD\%) & $15 \mathrm{mM} \mathrm{(3.6)}$ & $9 \mathrm{mM}(2.1)$ \\
& $45 \mathrm{mM}(2.1)$ & $30 \mathrm{mM}(1.9)$ \\
& $75 \mathrm{mM}(1.9)$ & $50 \mathrm{mM}(0.8)$
\end{tabular}

\footnotetext{
${ }^{\mathrm{a}}$ Mean $(n=5)$.
} 
frequency was chosen for all subsequent measurements.

The influence of square-wave amplitude on the peak current was studied in the range $10-100 \mathrm{mV}$. The peak current increased until a maximum at 50 $\mathrm{mV}$ and then remained constant. The square-wave amplitude of $50 \mathrm{mV}$ was adopted for the analysis.

With the optimum experimental conditions selected the peak current was linearly dependent on tramadol concentration.

\subsubsection{Evaluation of the method}

Standard calibration curves for tramadol hydrochloride were constructed (Fig. 4). Good linearity was obtained over the range $15-75 \mathrm{mM}$ (Table 1). The LOQ and LOD, calculated according USP 25 guidelines [13], were 7.3 and $2.2 \mathrm{mM}$, respectively. Precision assays were carried out at three levels $(15,45$ and $75 \mathrm{mM})$ and the results were good; the RSD\% values obtained ranged from 1.4 to $2.3 \%$ for repeatability (intra-day precision) and from 1.9 to $3.6 \%$ for intermediate (inter-day) precision (Table 1).

\subsection{Development of the flow injection method}

A single channel manifold was set-up (Fig. 2) and was afterwards gradually optimised with the purpose to allow the introduction of samples without pre-treatment and maximise both the reproducibility and sampling rate. Hence, the influence of several parameters such as the working electrode potential, flow rate $(Q 1)$, injection volume $(A)$ and the reactor length $(R)$ were studied using a $10 \mathrm{mM}$ solution of tramadol.

\subsubsection{Working electrode potential}

To optimise the working electrode potential a study of the variation of the peak height with the potential applied between 0.8 and $1.2 \mathrm{~V}$ was carried out. The peak height increased up to 1.0 $\mathrm{V}$ and then reached a plateau over the range 1.0-, $1.2 \mathrm{~V}$. Beyond $1.0 \mathrm{~V}$ a decrease in the reproducibility of the recorder output was observed. Therefore, the value of $1.0 \mathrm{~V}$ was kept constant for the subsequent measurements.

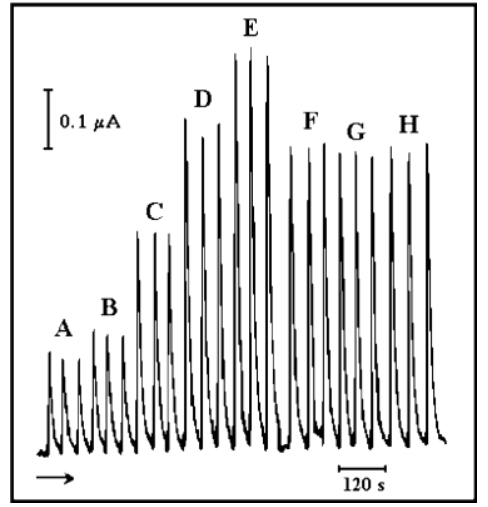

Fig. 5. Recorder output for a series of tramadol standards in $\mathrm{mM}$ ((A) 9.6, (B) 12.5, (C) 24.0, (D) 36.5, (E) 48.0) and real samples $(F, G, H)$ under the conditions described for the FIA technique.

\subsubsection{Flow rate}

Optimisation of the flow rate was dependent on the characteristics of the wall-jet cell whose small dead volume ("'1 ml [14]) does not allow the use of very high flow rates. The results obtained showed that a flow rate of $2.4 \mathrm{ml} \mathrm{min}^{-1}$ at the entry of the detector is the most adequate since higher flows produce high pressures within the system leading to less reproducible analytical signals. Although lower values led to reproducible signals the sampling rate was diminished. As a compromise between reproducibility and sampling rate a flow rate of $2.4 \mathrm{ml} \mathrm{min}^{-1}$ was selected.

\subsubsection{Injection volume}

To establish the most adequate injection volume, loops with lengths between 6 and $20 \mathrm{~cm}$ were made, using the same Teflon tubing used for the other parts of the system ( $0.8 \mathrm{~mm}$ i.d.), and tested. The use of loop lengths lower than $10 \mathrm{~cm}$ led to less reproducible signals whereas for higher lengths the sampling frequency was reduced. A $10 \mathrm{~cm}$ loop was therefore selected for the analysis. The sample volume corresponding to the chosen loop was accurately determined by titration of a solution of known concentration and this corresponded to 80 $\mathrm{ml}$ [15]. 
Table 2

Determination of tramadol in commercial pharmaceutical preparations using the SWV method and FIA with amperometric detector method (FIA-EC)

\begin{tabular}{llll}
\hline Formulation & Tramadol nominal content & SWV $^{\mathrm{a}}$ & FIA-EC $^{\mathrm{a}}$ \\
\hline Tramadol Asta Medica MG $_{\text {Tramadol Irex }}^{\dagger}$ & $50 \mathrm{mg} \mathrm{ml}^{-1}$ & 50.790 .8 & 49.890 .3 \\
Tramal $^{\dagger} 100$ & $50 \mathrm{mg} \mathrm{ml}^{-1}$ & 49.490 .6 & 49.090 .5 \\
Tramal ciclum MG $_{\text {Tramadol Asta Medica MG }}$ & $50 \mathrm{mg} \mathrm{ml}^{-1}$ & 50.590 .4 & 50.490 .3 \\
Tramal $^{\dagger}$ & $100 \mathrm{mg} \mathrm{ml}^{-1}$ & 100.090 .9 & 101.690 .8 \\
Travex $^{\dagger}$ & $100 \mathrm{mg} \mathrm{ml}^{-1}$ & 97.590 .6 & 100.790 .4 \\
Paxilfar $^{\dagger}$ & $50 \mathrm{mg} \mathrm{capsule}^{-1}$ & 50.090 .9 & 50.290 .2 \\
\end{tabular}

${ }^{\text {a }}$ Mean and standard deviations of three determinations for different samples (mg tablet ${ }^{-1}$ or $\mathrm{mg} \mathrm{ml}^{-1}$ ).

\subsubsection{Reactor length}

The optimisation of the reactor length $(R)$, in which the mixture of the sample plug and electrolyte takes place, was also carried out. Different reactor lengths were tested, between 20 and $45 \mathrm{~cm}$. The results showed less reproducible signals for lengths smaller than $35 \mathrm{~cm}$ due to insufficient mixing. Although higher lengths led to reproducible signals there was an unnecessary dilution of the sample and a decrease of the sampling rate. Hence, a $35 \mathrm{~cm}$ reactor length was adopted for subsequent analysis.

\subsubsection{Evaluation of the method}

The flow injection methodology developed led to reproducible results (Fig. 5). Using the optimised parameters a calibration curve was set up with standard solutions in the $9-50 \mathrm{mM}$ concentration range and good linearity was obtained (Table 1). The LOQ obtained was $5.7 \mathrm{mM}$ and the LOD was $1.7 \mathrm{mM}$. Precision assays were carried out at three levels $(9,30$ and $50 \mathrm{mM})$ and the results were very good; RSD\% values ranged from 0.6 to $1.2 \%$ for repeatability and from 0.8 to $2.1 \%$ for intermediate precision (Table 1). This manifold enables sampling rates of about 90 samples $\mathrm{h}^{-1}$.

\subsection{Analytical application to pharmaceutical dosage forms}

In order to assess the usefulness of the methods they were applied to the determination of trama- dol in eight pharmaceutical dosage forms available in Portugal.

For the voltammetric method, square-wave voltammograms were recorded for different tramadol standards in the concentration interval 15 to $75 \mathrm{mM}$ (Fig. 4) and for the commercial samples. The flow injection system was calibrated by three replicate injections of five tramadol stan- dards in a concentration range between 9 and $50 \mathrm{mM}$. Fig.

5 shows the diagram, corresponding to three replicate injections of five standard solutions and three samples, obtained in the determination of tramadol hydrochloride in pharmaceutical preparations.

In Table 2 the mean results and the corresponding standard deviation for three replicate determinations for each dosage form are presented. The concentration values found were in good agreement with the labelled amounts.

Accuracy was assessed by means of recovery assays at three different concentrations $(15,35$ and $60 \mathrm{mM}$ for voltammetric method and 9, 25 and 40 $\mathrm{mM}$ for flow injection method); recoveries ranged from 97 to $102 \%$ revealing a good accuracy of the results.

A systematic quantitative study was undertaken to verify the effect of different additives and excipients, which may be associated with tramadol in the formulations tested, using the developed methods. No significant interference was observed from the excipients commonly used such as sodium acetate, potassium sorbate, lactose and sacarose up to 10 -fold. These results show that 
these methods are applicable to the pharmaceutical preparations available commercially.

\section{Conclusions}

The electrochemical methods developed for the quantification of tramadol have proved to be a good alternative and advantageous over the reported analytical methods due to their sensitivity, rapidity and accuracy. The good recoveries and low relative standard deviation reflect the high accuracy and precision of the proposed methods. Moreover, the methods are simple, easy to operate and inexpensive making them an excellent tool for the routine determination of tramadol in quality control laboratories. The flow injection system enables the determination of tramadol in pharmaceutical preparations at a rate of 90 samples $\mathrm{h}^{-1}$.

\section{Acknowledgements}

We thank Tecnifar (Lisbon, Portugal) for the generous gift of tramadol hydrochloride.

\section{References}

[1] L.J. Scott, C.M. Perry, Drugs 60 (2000) 139 -176.

[2] M. Valle, J.M. Pa'von, R. Calvo, M.A. Campanero, I.F. Trocóniz, J. Chromatogr. B 724 (1999) 83-89.

[3] M. Nobilis, J. Kopecky, J. Kvetina, J. Chladek, Z. Svoboda, V. Vorisek, F. Perlik, M. Pour, J. Kunes, J. Chromatogr. A 949 (2002) 11-/22.

[4] W.C. Sung, S.H. Chen, Electrophoresis 22 (2001) 4244-, 4248.

[5] U.B. Soetebeer, M.O. Schierenberg, H. Schulz, P. Andresen, G. Blaschke, J. Chromatogr. B 765 (2001) 3-13.

[6] J. Rajput-Sadhana, D. Trivedi-Priti, Indian Drugs 38 (2001) 100-101.

[7] H.E. Abdellatef, J. Pharm. Biomed. Anal. 29 (2002) 835 842.

[8] I.Y. Zaghloul, M.A. Radwan, J. Liq. Chromatogr. Relat. Technol. 20 (1997) 779 -787.

[9] M. Pospisilova, M. Polasek, V. Jokl, J. Pharm. Biomed. Anal. 18 (1998) 777-783.

[10] J.L. Yue, Q.H. Yu, D.H. Li, G.Z. Zhao, W. Shi, L.R. An, Fenxi Huaxue 28 (2000) 84 -86.

[11] S. Alegret, J. Alonso, J. Bartroli, A.A.S.C. Machado, J.L.F.C. Lima, J.M. Paulis, Quim. Anal. 6 (1987) 278 -294.

[12] A.M. Oliveira Brett, M.M.M. Grazina, T.R.A. Macedo, C. Oliveira, D. Raimundo, J. Pharm. Biomed. Anal. 11 (1993) 203-206.

[13] U.S. Pharmacopoeia, 25 Edition, United States Pharmacopoeial Convention, Rockville, 2002, p. 2256.

[14] Electrochemical Detection in HPLC, Metrohm, Herisau, 1984.

[15] J.L.F.C. Lima, A.O.S.S. Rangel, J. Int. Sci. Vigne. Vin. 24 (1990) 49-61. 